\title{
Refinement with multipolar scattering factors
}

\author{
B.Gruza, M. Chodkiewicz and P.M.Dominiak \\ Biological and Chemical Research Centre, Department of Chemistry, University of Warsaw, Poland \\ b.gruza@uw.edu.pl
}

Electron diffraction makes it possible to obtain crystal structures at atomic resolutions, for both small and macro-molecules [1-2]. For this purpose, the same as in case of X-ray diffraction, it is necessary to use scattering factors model. After years of experience people learned that Independent Atom Model (IAM) is not the best choice for X-ray diffraction. It is not a surprise that also for electron diffraction it will not give the best results. Different aspherical models, already known for X-ray diffraction, can be implemented for electron diffraction. However, it is necessary to investigate their possibilities and limits, to verify correctness of obtained structures.

We present analysis of refinements of Transferable Aspherical Atom Model (TAAM) with parameters of multipolar model taken from MATTS databank (databank of Multipolar Atom Types from Theory and Statistical clustering) - successor of UBDB databank [3]. We used electron scattering factors implemented in DiSCaMB library[4] and interfaced with Olex2-1.3[5]. Such solution is available through .tsc files [6] and it requires little effort (Fig.1).

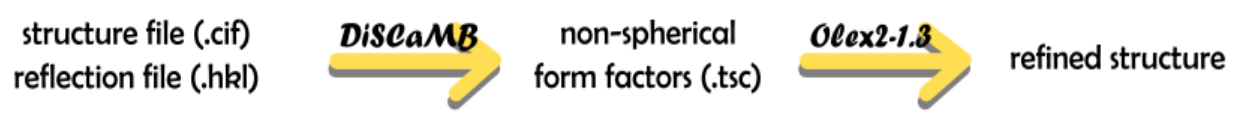

Figure 1. General refinement procedure

Numerous refinements were performed against experimental electron structure factors and theoretical electron structure factors so as to find optimum refinement strategy. We discuss, inter alia, possibility of refinement of atomic displacement parameters, both for hydrogen and non-hydrogen atoms or positions of hydrogen atoms. It is interesting how they change e.g. with resolution cut-off. To confirm that our conclusions could be transferred for different, but still organic structures, we made simulations for several pharmaceutical compounds, such as carbamazepine, paracetamol, 1-methyluracil.

[1] Clabbers, MTB; van Genderen, E; Wan, W; Wiegers, EL; Gruene, T; Abrahamsa, JP. (2017). Acta Cryst. D Struct Biol. 73, 738-748.

[2] Jones, CG; Martynowycz, MW; Hattne, J; Fulton, TJ; Stoltz, BM; Rodriguez, JA; Nelson, HM; Gonen, T. (2018). ACS Cent. Sci. 4(11), $1587-1592$.

[3] Jarzembska, KN; Dominiak. PM. (2012). Acta Cryst A, A68, 139-147.

[4] Chodkiewicz, ML; Migacz, S; Rudnicki, W; Makal, A; Kalinowski, JA; Moriarty, NW; Grosse-Kunstleve, RW; Afonine, PV; Adams, PD; Dominiak, PM. (2018). J. Appl. Cryst., 51, 193-199.

[5] Dolomanov, OV; Bourhis, LJ; Gildea, RJ; Howard, JAK; Puschmann, H. J. (2009). Appl. Cryst., 42, 339-341.

[6] Midgley, L., Bourhis, L.J., Dolomanov, O., Peyerimhoff, N., Puschmann, H. (2019). https://arxiv.org/abs/1911.08847v1

Keywords: aspherical scattering factors, TAAM, electron diffraction, structure refinement

Support of this work by the National Centre of Science (Poland) through grant OPUS No.UMO-2017/27/B/ST4/02721 and PL-Grid through grant ubdb2019 are gratefully acknowledged. 